\title{
The Giraffe as a Natural Animal Model for Resistance to Heart Failure with Preserved Ejection Fraction
}

B. N. Horowitz, ${ }^{1,2,3^{*}}$ B. M. Baccouche, ${ }^{2,4}$ Tejas Shivkumar, ${ }^{5}$ Mads Frost Bertelsen, ${ }^{6}$ Christian Aalkjær, ${ }^{7}$ Morten H. Smerup, ${ }^{8}$ Olujimi A. Ajijola, ${ }^{9}$ Joseph Hadaya, ${ }^{9}, 10$ Tobias Wang ${ }^{11}$

${ }^{1}$ Department of Medicine, Harvard Medical School, Boston, Massachusetts, USA

${ }^{2}$ Department of Human Evolutionary Biology, Harvard University, Cambridge, Massachusetts, USA

${ }^{3}$ Division of Cardiology, David Geffen School of Medicine at UCLA, Los Angeles, CA, USA

${ }^{4}$ Department of Public Health and Primary Care, University of Cambridge, Cambridge, United Kingdom

${ }^{5}$ Brentwood School, Los Angeles, California, USA

${ }^{6}$ Copenhagen Zoo, Frederiksberg, Denmark

${ }^{7}$ Department Biomedicine, Aarhus University, Aarhus, Denmark

${ }^{8}$ Department of Cardiothoracic Surgery, Copenhagen University Hospital, Rigshospitalet, Copenhagen, Denmark

${ }^{9}$ UCLA Cardiac Arrhythmia Center, David Geffen School of Medicine at UCLA, Los Angeles, CA, USA

${ }^{10}$ Molecular, Cellular and Integrative Physiology Program, UCLA

${ }^{11}$ Zoophysiology, Department of Biology, Aarhus University, Aarhus, Denmark

*Corresponding Author

Barbara N. Horowitz

Department of Human Evolutionary Biology

Harvard University

11 Divinity Avenue

Peabody Museum, Room 47

Cambridge, MA 02138, USA

Tel: 1-310-413-8131

E-mail: natterson-horowitz@fas.harvard.edu

ORCID: 0000-0002-6145-9689

Basil M. Baccouche

Department of Public Health and Primary Care

University of Cambridge

Strangeways Research Laboratory

Wort's Causeway

Cambridge, CB1 8RN, United Kingdom

ORCID: 0000-0002-8840-5429

Tejas Shivkumar

Brentwood School

522 Cashmere Terrace

Los Angeles, CA 90049

ORCID: 0000-0002-9181-9702 
Mads Frost Bertelsen

Copenhagen Zoo

Roskildevej 38, DK-2000

Frederiksberg, Denmark

ORCID: 0000-0001-9201-7499

Christian Aalkjær

Dept Biomedicine, Aarhus University

Høegh-Guldbergs Gade 10, 8000

Aarhus C, Denmark

ORCID: 0000-0003-2618-4015

Morten Smerup

Department of Cardiothoracic Surgery, Copenhagen University Hospital, Rigshospitalet Østbanegade 113, 1. tv.

2100 Copenhagen, Denmark

ORCID: 0000-0002-4350-3682

Olujimi A. Ajijola

UCLA Cardiac Arrhythmia Center

David Geffen School of Medicine at UCLA

100 Medical Plaza, Suite 660

Los Angeles, CA 90095

ORCID: 0000-0001-6197-7593

Joseph Hadaya

UCLA Cardiac Arrhythmia Center

David Geffen School of Medicine at UCLA

100 Medical Plaza, Suite 660

Los Angeles, CA 90095

ORCID: 0000-0001-9902-0578

Tobias Wang

Zoophysiology, Department of Biology

Aarhus University, Aarhus, Denmark

ORCID: 0000-0002-4350-3682

ABSTRACT WORD COUNT: 144

TEXT WORD COUNT: 2422

FIGURES: 3

EXPLANATORY BOXES: 3 


\begin{abstract}
Heart failure with preserved ejection fraction (HFpEF) is a leading form of human cardiovascular disease and commonly associated with systemic hypertension. Unique evolved adaptations in giraffe myocardia may be a natural animal model of resistance to HFpEF. In humans, pressureoverload induced left ventricular thickening (PLVT) impairs diastolic relaxation, elevates left atrial pressures and may progress to heart failure with symptoms including exercise intolerance. In healthy giraffe, the left ventricle thickens as developmental neck lengthening widens the vertical distance between the heart and head increasing pressures needed to maintain constant brain perfusion. Yet, diastolic relaxation and exercise capacity are unimpaired, a critical adaptation for prey species such as giraffe. The proximate mechanisms underlying this unique cardiovascular physiology are not yet characterized. Developmental PLVT in giraffe emerges as a species-specific evolved adaptation which offers a roadmap for identifying innovations in therapeutic and prevention strategies for HFpEF.
\end{abstract}

\title{
INTRODUCTION
}

Bioinspired strategies have sparked a rapidly growing list of diagnostic and therapeutic applications including wound healing, anticoagulation, infection control and biomedical imaging [1-4]. However, there have been few efforts to apply bioinspired approaches to human heart disease, especially heart failure. The purpose of this review is to: 1) define the unique cardiovascular adaptations of the modern giraffe as a potential natural animal model of resistance to heart failure with preserved ejection fraction (HPpEF) and 2) demonstrate how expanded physician awareness in comparative animal physiology can serve to inspire innovations to solve complex clinical human pathophysiology.

\section{Heart Failure with Preserved Ejection Fraction (HFpEF)}

Cardiovascular disease (CVD) is the leading cause of death in the United States, killing a person every 37 seconds [5]. Heart failure (HF), a chronic progressive form of CVD, is characterized by the heart's inability to pump sufficient blood to meet needs and requirements of the body. Among patients over 65 years of age in the US, it is the leading reason for hospitalization and its prevalence around the world is increasing to due to an aging population [6-8]. Heart failure is commonly classified based on whether the left ventricular ejection fraction $(E F)$ is in a normal range, borderline, or reduced [9]. Advances in both pharmacologic and device-based therapies over the past four decades have significantly lowered mortality and morbidity in patients with reduced left ventricular EF [10]. Yet, despite significant research investment, similar progress has yet to be accomplished in the treatment of heart failure with preserved ejection fraction (HFpEF), commonly referred to as "diastolic heart failure". The human costs are high: HFpEF accounts for half of all heart failure diagnoses and has an estimated five-year survival rate of only $35 \%$ [11]. While multiple conditions including obesity, coronary artery disease, diabetes, and chronic kidney disease are associated with $\mathrm{HFpEF}$, systemic hypertension is one of the most common and presumably contributing factors [12].

Arterial hypertension follows Ohm's law for fluids as a response to pathologically increased arterial resistance in order to maintain cardiac output. However, increasing afterload imposes a greater workload on the left ventricular myocardium leading to hypertrophic changes. Progression to heart failure syndromes in parallel with other problems that include stroke, arterial disease, coronary events, and general cardiovascular mortality often follow [13]. Hypertension has reached epidemic 
proportions over the past three decades. Given its connection to left ventricular hypertrophy (LVH), it is not surprising that HFpEF has emerged as the fastest growing form of human heart failure [14].

\section{Pathological PLVH (Left Ventricular)}

Like any muscle, the left ventricle responds with hypertrophy when exposed to increased workload, but the increase in left ventricular thickness is a double-edged sword. LVH is adaptive and compensatory in its early stages, providing the systolic force needed to maintain cardiac output while keeping a normal wall tension [15]. However, this initial adaptive response alters the physical and functional properties of the human ventricle. Biochemical signaling, including the activation of cytosolic and nuclear messengers in response to hypertrophic stimulus to the cardiomyocyte, affects the morphologic alterations associated with LVH by regulating gene expression. The resulting physical alterations include cardiomyocyte longitudinal and cross-sectional growth and an increase in interstitial and perivascular connective tissue [16, 17].

The left ventricular pressure of a normal, non-hypertrophied heart rises to a peak during systole and fall to its nadir during diastole. Because the pulmonary circulation is "exposed" to the left ventricle during diastole, a low pressure at end diastole "protects" the lungs from excess volume and pressure which could impact oxygenation [18]. In HFpEF patients, left ventricular diastolic pressures fail to fall to physiologically normal levels, leading to increased left atrial and pulmonary pressures. The exposure of pulmonary vasculature to elevated pressures leads to pulmonary edema with clinical consequences including progressively reduced exercise tolerance, hypoxia, and in some cases, even death [19].

Numerous recent therapies developed to counter HFpEF have been largely unsuccessful in delivering the robust clinical benefits needed by the rapidly increasing population of patients with HFpEF. The reduction in mortality and morbidity that device-based and pharmacologic therapies have provided to patients with systolic heart failure do not yet exist for HFpEF patients [20] and the lack of suitable animal models have been identified as one of the primary obstacles for therapeutic innovation in HFpEF [21].

\section{GIRAFFE: A NATURAL ANIMAL MODEL}

\section{Developmental Physiologic PLVT}

Species-specific cardiovascular adaptations found in Giraffa camelopardalis, the modern giraffe, may represent a naturally occurring model of resistance to HFpEF.

At birth, the thickness of the giraffe ventricle is comparable to what has been observed in other young mammals [22]. Over the course of development and general somatic growth, the lengthening of the neck increases the vertical distance between the heart and the brain, requiring systolic blood pressures of 2-300 $\mathrm{mmHg}$ at heart level to maintain cerebral perfusion. While dangerously hypertensive by non-giraffe mammalian standards, these pressures are normal and crucial for a giraffe's hemodynamics performance [22-28].

As afterload increases with somatic growth, the giraffe's left ventricle thickens to alleviate increased wall stress as the Law of Laplace would predict. In humans with systemic hypertension, the left ventricle also thickens to maintain normal wall stresses despite increasing afterload (Figure 1). Progressive thickening reduces the relative size of the ventricular cavity [30]. Deposition of interstitial fibrosis leads to increased ventricular stiffness (reduced compliance). Studies of mammalian heart structure reveal highly conserved, patterned relationships between left ventricular 
wall thickness and cavity size. In modern giraffes, this relationship is uniquely altered although a reduced stroke volume consistent with reduced cavity size is present.
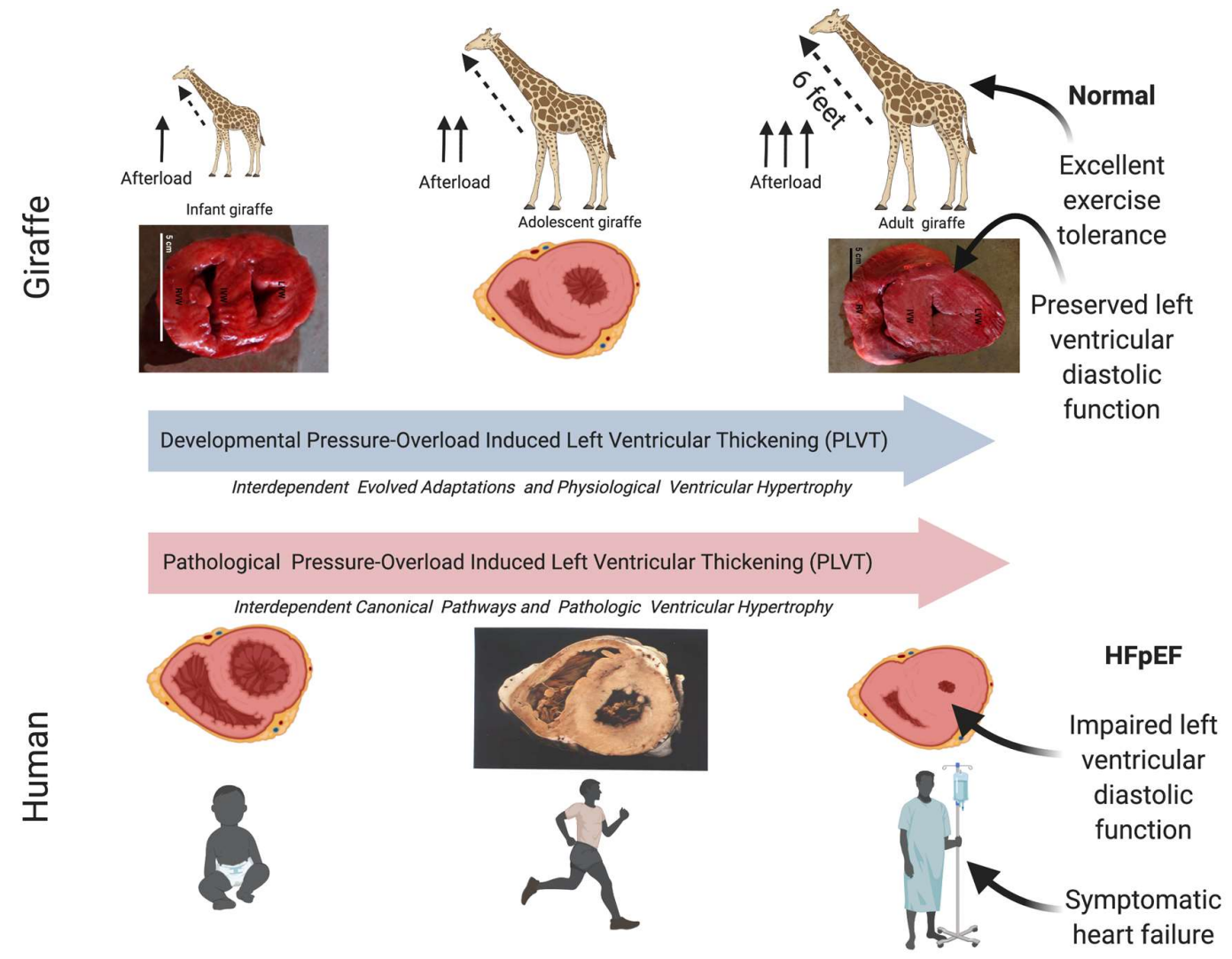

Figure 1. Comparison of the giraffe ventricular response to developmental PLVT vs. the human ventricular response to chronic hypertension-induced left ventricular thickening. Hypertensioninduced left ventricular PLVT in humans leads to heart failure (HFpEF). Developmental PLVT in growing giraffes does not compromise exercise capacity, an important adaptation for a prey species.

As shown in the phylogeny (Figure 2), giraffes and okapi diverged from their shared common ancestor 10-12 million years ago, and it is over this period of time that the iconic long neck of the giraffe evolved [29]. 


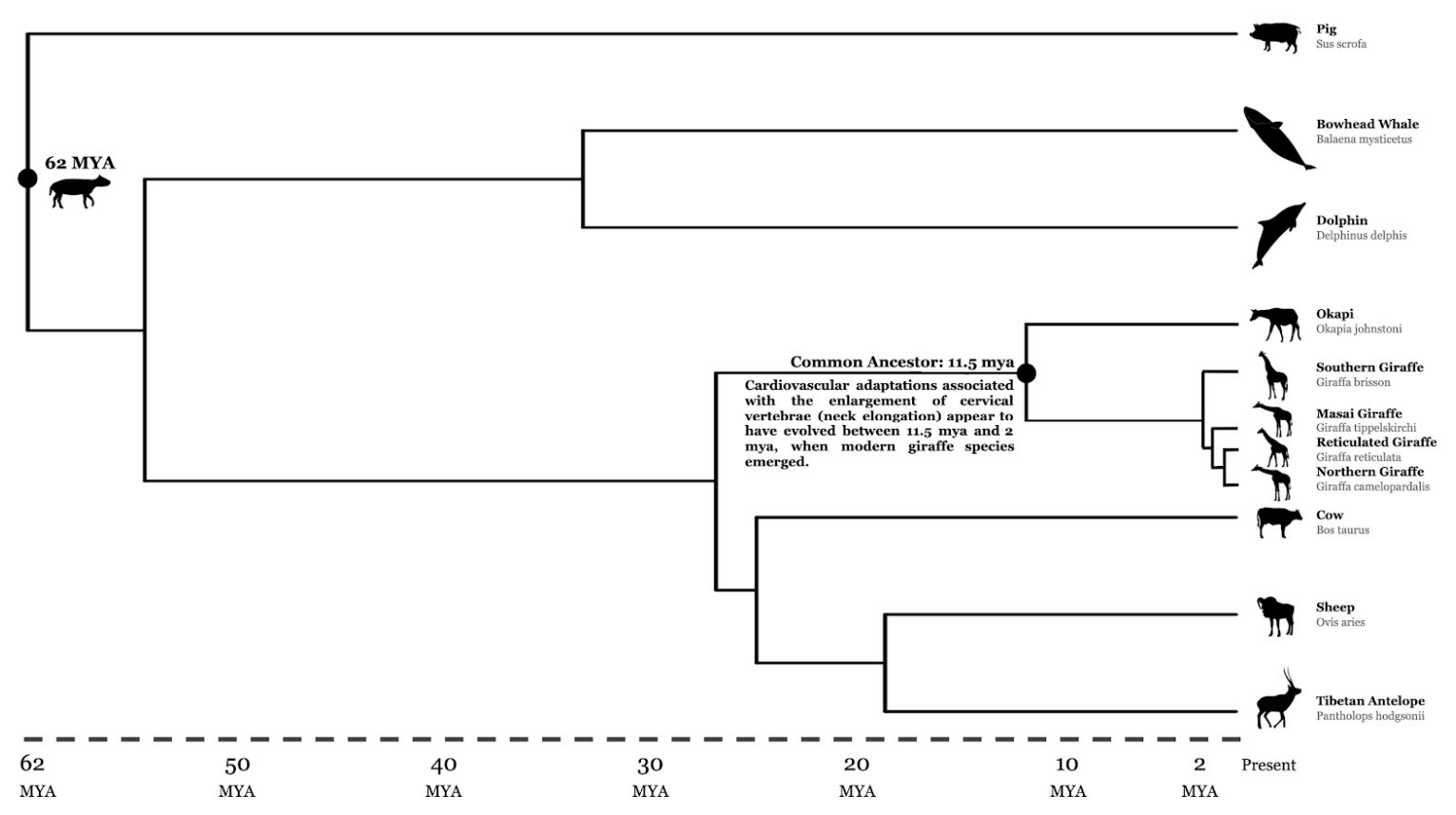

Figure 2. The divergence of the giraffe from the okapi and other mammals. Made using the Interactive Tree of Life [31].

The proposed fitness-enhancing benefits of the long neck include greater access to high foliage, enhanced predator detection, and sexual selection [32]. But, the survival and reproductive benefits of a longer neck also introduced a unique physiological challenge for the adult giraffe cardiovascular system, namely the obstacle of providing adequate cerebral perfusion of a brain situated over two meters above the left ventricle. The adaptations sparked by this hemodynamic challenge are the core of what may be a new approach to HFpEF.

The gross left ventricular morphology of the modern giraffe shares some but not all characteristics found in other mammals [33-35]. For example, the size and mass of the giraffe heart itself are similar to what is found in similarly-sized mammals. However, relative to other species, the volume of blood contained in the giraffe's left ventricular cavity is notably smaller, especially compared with the thickness of its ventricular wall $[26,27]$. The uniquely high ratio between left ventricular wall thickness and cavity size in the adult giraffe is thus a highly notable deviation; a human with a comparable ratio would be highly symptomatic with a poor clinical prognosis.

\section{Exercise Intolerance vs. Tolerance}

Humans with advanced LVH often experience exercise limitations and the disease symptoms often associate with physical activity. Cellular, structural and functional alterations induced by LVH compromise the ability of the left ventricle to relax during the diastolic phase of the cardiac cycle [36]. This effect is exacerbated in physical activity when heart rates rise and diastolic filling times are shortened. The severe symptoms and functional limitations in patients with advanced HFpEF are often resistant to pharmacologic intervention. The heart failure syndromes associated with hypertension are considered unavoidable consequences of ventricular hypertrophy. 
The developmental pressure-induced left ventricular wall thickness increase (PLVT) of the giraffe heart has special relevance for HFpEF because it is an example of a mammalian heart where PLVT does not appear to impact exercise capacity. The hemodynamic consequences of pathological left ventricular PLVT (human heart failure patient) versus developmental PLVT (normal giraffe) are shown in (Figure 3).

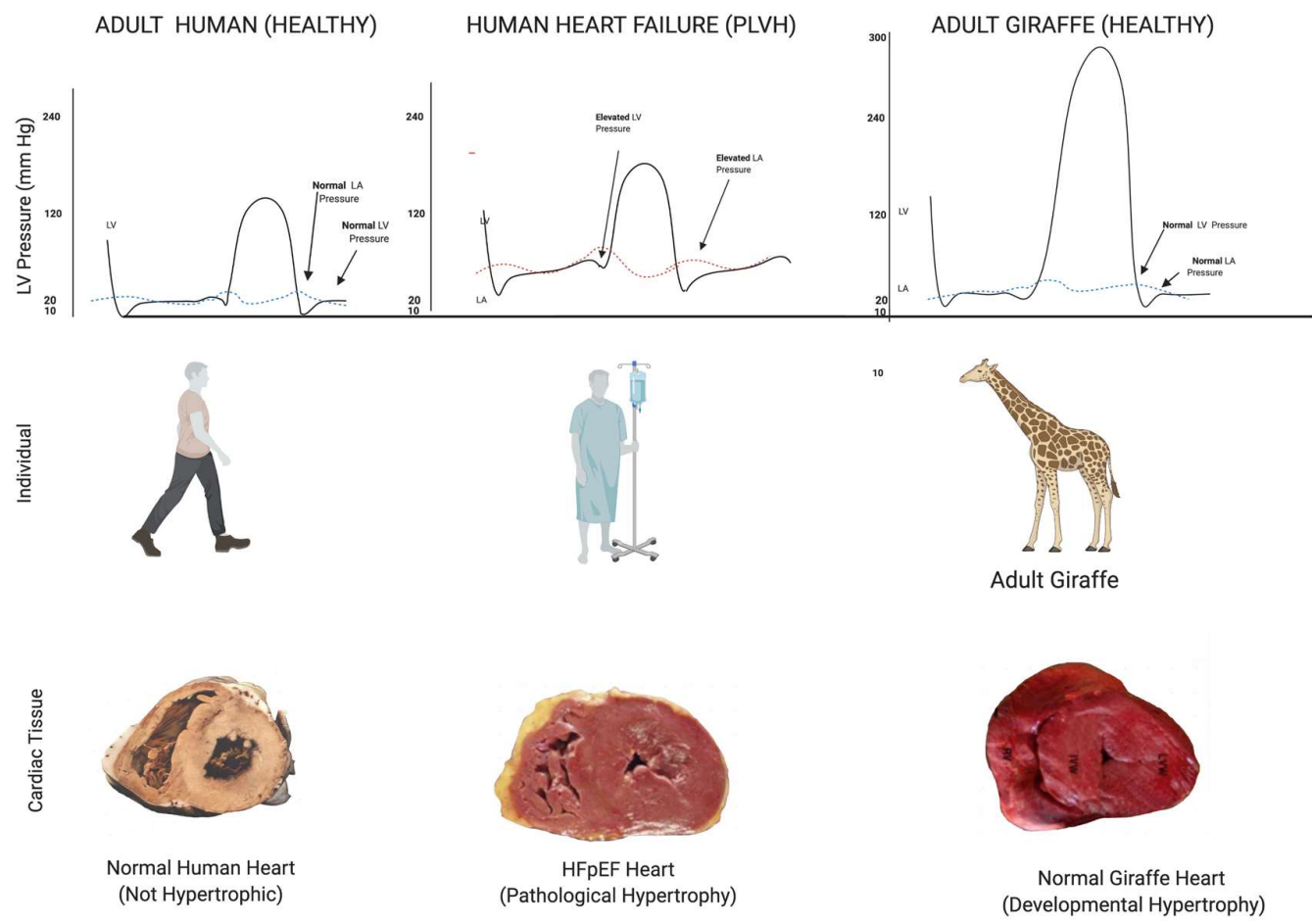

Figure 3. Comparison of healthy human adult, human heart failure, and healthy adult giraffe hearts in terms of gross ventricular anatomy and left atrial and ventricular pressure relationships. Despite significant PLVH left ventricular thickening giraffe cardiac pressures do not show the elevated left atrial and ventricular pressures seen in humans with severe PLVH. Giraffe ventricular pressures adapted from Smerup et al., 2016 [27].

The absence of exercise intolerance in giraffe points to unique adaptations allowing their hearts to maintain normal wall stress in the face of rising afterload through myocardial wall thickening without compromising diastolic relaxation and exposing pulmonary circulation to increased pressure. Maintaining superb exercise capacity is of clear importance in a prey species like the giraffe who must flee predators who chase them at speeds as high as $60 \mathrm{~km} / \mathrm{hr}$ [37]. In a particularly impressive study, heart rates up to $170 \mathrm{~min}^{-1}$ were measured by radiotelemetry in freely moving giraffes as they were running to avoid capture. During flight from predators increased myocardial oxygen demand contributes to rising heart rates which reduces relative diastolic filling times. Although hemodynamic measurements of giraffes exercising at maximum capacity are not presently available, the importance of fleeing for a prey species is self-evident [25].

\section{Simulated Exercise: Emerging Support for Hypothesis}

Recently an unpublished investigation measured left ventricular pressures during a pharmacologically-induced simulation of predator-evasion autonomic physiology. Progressive 
infusion of norepinephrine was administered to mimic the heart rates and blood pressures previously measured in fleeing giraffe, a condition associated with high sympathetic tone. This protocol raised heart rates and blood pressures to levels which exceeding Van Critters' measurements in exercising giraffes [25]. Heart rates rose from $27 \mathrm{~b} / \mathrm{m}$ at rest to $118 \mathrm{~b} / \mathrm{m} 3$ minutes and $150 \mathrm{~b} / \mathrm{m}$ six minutes into the infusion. Blood pressures rose three-fold from $167 \mathrm{mmHg}$ at baseline to over $500 \mathrm{mmHg}$ at peak infusion. Of note was the absence of appreciable elevation in left ventricular pressure during diastole despite tachycardia and extremes of afterload. This finding supports the hypothesis that evolved adaptive mechanisms preventing diastolic compromise at extremes of afterload and heart rates protect this prey species from fitness-reducing exercise intolerance seen under similar conditions in humans with PLVT.

\section{Potential Mechanisms}

The cellular and subcellular mechanisms that protect giraffe hearts from the adverse hemodynamic consequences of PILT remain largely unidentified and characterized. However, unique mutations in the modern giraffe genome point to fibrosis suppression as a possible mechanism. In humans, interstitial fibrosis contributes to ventricular stiffening and subsequent diastolic impairment in HFpEF. While numerous interdependent systems are involved in the deposition of collagen and extracellular matrix proteins in myocardial fibrosis, the renin-angiotensin system (RAS) plays a central role in this cascade [38]. Angiotensin-converting enzyme (ACE), a key enzyme in the RAS, converts decapeptide angiotensin I to octapeptide angiotensin II, which has numerous effects on vascular and myocardial tissue. Notably, angiotensin II plays an important role in hypertensioninduced fibrosis [39-41].

An observed relative absence of fibrosis in giraffe myocardia (in comparison with similarly thickened human ventricles) may be linked to the unique mutation in the ACE genes of the giraffe genome [25]. A comparison of the giraffe genomes and the genome of its closest living evolutionary relative, the okapi, found 70 genes with "multiple signs of adaptation" not seen in other eutherian mammals [29]; five of these genes are found within the developmental pathways that lead to cardiac fibrosis [42]. Preliminary data from reviewing 136 necropsy reports suggests a reduced propensity for myocardial fibrosis in the giraffe relative to humans and other mammalian species [42].

Because fibrosis impairs ventricular diastolic relaxation in humans, potential fibrosis inhibition mechanisms in the giraffe may underlie its preserved exercise capacity. There are undoubtedly multiple interdependent mechanisms involved in the unique diastolic physiology of these animals. It is currently unclear how differences in autonomic regulation, myocardial innervation, neuroendocrine function and other factors contribute to a giraffe's unique exercise capacity. Far clearer is what the modern giraffe sprinting on the savannah represents to the field of human heart failure: a high-performance model of preserved exercise capacity in the presence of significant PILVT. 


\section{RECOGNIZING EVOLUTIONARY ADAPTATIONS AS A SOURCE OF THERAPEUTIC INNOVATIONS}

The giraffe's unique physiology has long been a source of fascination to biologists and physiologists. Goetz and Keen, two of the first to gather concrete physiological data on the giraffe noting its 'high' blood pressures, by human standards. Others have studied the giraffe's resistance to orthostatic changes with shifts in neck position and the ability of its kidneys to withstand high arterial pressures [23, 43-55]. Earlier studies show thick ventricular walls in the giraffe [23, 27]. Smerup et al. studied the giraffe's ability to generate and sustain high afterload investigating myocardial architecture, cellular structure and hemodynamics [27]. Their findings included a left ventricular pressure time curve demonstrating normal diastolic ventricular pressures. While the animal was under anesthesia and not tachycardic, normal diastolic pressures would not be predicted in a morphologically comparable ventricle.

The importance of this finding to human cardiovascular medicine is significant. The existence of a mammalian cardiovascular system in which progressive PLVT is not accompanied by reduced diastolic relaxation or elevated cardiopulmonary pressures disrupts conventional paradigms. It directs attention away from pharmacologic therapies directed at altering lusitropic characteristics of myocardial tissue and towards developmental pathways and related regulatory systems.

Within the field of cardiovascular medicine, numerous species exhibit physiologic characteristics with salience for human health. Okapi are uniquely vulnerable to peripartum cardiomyopathy, a heart failure syndrome associated with late pregnancy in women [56]. Some avian taxa, parrots and pigeons, develop significant atherosclerosis in captive settings more than others [57]. Groups of rock kangaroos with increased ventricular thickening have been noted to have elevated sudden death risk suggesting the species may be a natural animal model for hypertrophic cardiomyopathy, the leading cause of cardiovascular death in young human athletes [58]. Many other species exhibit unique sets of cardiovascular physiologies with relevance to a wide range of human disorders. Some

Given the salience of PILT in the giraffe other species-specific cardiovascular characteristics to human health, why are connections relatively unexplored? One factor has been the limited extent to which physicians perceive the natural world as a source of insights for complex human pathophysiology. Amongst veterinarians and wildlife biologists, training in comparative physiology, emphasizing differences between species and seeking to define general principles, is a core discipline that underpins any understanding of how animals interact with their environment. Yet, modern medical education does not traditionally include broad instruction on the diverse range of high-performance physiologies of other species. Greater collaborative interactions between physicians, veterinarians, animal physiologists and wildlife biologists would increase the likelihood that biomedical investigators would identify a 'solution' to challenging human pathophysiologies in the natural world.

Rudolf Virchow, father of modern pathology, observed that, "Between human and animal medicine there is no dividing line" [59]. Despite Virchow's early insight, the separation between human, comparative and veterinary cardiology persist, and the lack of communication between these research fields impede innovations to the detriment of human cardiovascular disease. As physicians and investigators increasingly perceive biodiversity in the natural world as a source of insights for clinical medicine, bioinspired solutions to the most challenging cardiovascular issues may emerge. 


\section{FUNDING AND CONFLICT OF INTEREST: None}

ACKNOWLEDGEMENT: The authors wish to thank Susan Kwan for her technical assistance in preparing the manuscript for submission.

\section{REFERENCES}

1. Speck O, Speck T. An Overview of Bioinspired and Biomimetic Self-Repairing Materials. Biomimetics (Basel) 2019;4:26.

2. Wang $\mathrm{C}$, Wang $\mathrm{S}$, Pan $\mathrm{H}$, et al. Bioinspired liquid gating membrane-based catheter with anticoagulation and positionally drug release properties. Science Advances 2020;6. https://advances.sciencemag.org/content/6/36/eabb4700.

3. Yang G, Chen S, Zhang J. Bioinspired and Biomimetic Nanotherapies for the Treatment of Infectious Diseases. Frontiers in Pharmacology 2019. doi.org/10.3389/fphar.2019.00751

4. Larkin W, Etienne-Cummings R, Van Der Spiegel J. Bioinspired Imaging: Discover, Emulation, and Future Prospects. Proceedings of the IEEE 2014;102:1404-10.

5. Heron M. Deaths: Leading Causes for 2017. National Vital Statistics Report 2019;68:77.

6. Savarese G, Lund LH. Global Public Health Burden of Heart Failure. Card Fail Rev 2017;3:7-11. doi: 10.15420/cfr.2016:25:2

7. Bui AL, Horwich B, Fonarow GC. Epidemiology and risk profile of heart failure. Nature Reviews: Cardiology 2011;8:30-41.

8. Ferrari R, Balla C, Fucili A. Heart failure: An historical perspective. European Heart Journal Supplements 2016;18:G3-10.

9. Federmann M, Hess OM Differentiation between systolic and diastolic dysfunction. European Heart Journal 1994;15 (Supp D): 2-6.

10. Kitai T, Tang WW. Recent advances in treatment of heart failure. F1000Research 2015;4(F1000 Faculty Rev):1475.

11. Oktay AA, Rich JD, Shah SJ. The Emerging Epidemic of Heart Failure with Preserved Ejection Fraction. Current Heart Failure Reports 2013;10(4). https://doi:10.1007/s11897013-0155-7.

12. Lalande S, Johnson BD. Diastolic Dysfunction: A Link between Hypertension and Heart Failure. Drugs Today 2008;44:503-13.

13. Aronow WS. Hypertension and left ventricular hypertrophy. Annals of Translational Medicine 2017;5(15). https://doi.org/10.21037/atm.2017.06.14

14. World Health Organization. Raised blood pressure. https://www.who.int/gho/ncd/risk_factors/blood_pressure_prevalence_text/en/ (3 August 2020, date last accessed)

15. Nichols AB, Sciacca RR, Weiss MB et al. (1980). Effect of left ventricular hypertrophy on myocardial blood flow and ventricular performance in systemic hypertension. Circulation 1980;62:329-49.

16. Garcia JAD, Incerpi EK. Factors and mechanisms involved in left ventricular hypertrophy and the anti-hypertrophic role of nitric oxide. Arq Bras Cardiol 2008;90:409-16.

17. Marketou, ME, Parthenakis F, Vardas PE. Pathological Left Ventricular Hypertrophy and Stem Cells: Current Evidence and New Perspectives. Stem Cells Int 2016:5720758. doi: $10.1155 / 2016 / 5720758$ 
18. Thenappan T, Prins KW, Pritzker MR et al. The Critical Role of Pulmonary Arterial Compliance in Pulmonary Hypertension. Annals of the American Thoracic Society 2016;13:276-84.

19. Purvey M, Allen G. Managing acute pulmonary oedema. Australian Prescriber 2017; 40:59-63.

20. Oren O, Goldberg S. Heart Failure with Preserved Ejection Fraction: Diagnosis and Management. The American Journal of Medicine 2017;130:510-516. https://doi.org/10.1016/j.amjmed.2016.12.031

21. Roh J, Houstis N, Rosenzweig A. Why don't we have proven treatments for HFpEF? Circulation Research 2017;120:1243-5.

22. Mitchell G, Skinner. An allometric analysis of the giraffe cardiovascular system. Comp Biochem Physiol 2009;154:523-529.

23. Goetz RH, Warren JV, Gauer OH, et al. Circulation of the giraffe. Circ Res 1960;8:104958.

24. Elsner R, Franklin DL, Van Citters RL, Kenney DW. Cardiovascular Defense against Asphyxia. Science 1966;153:941-49.

25. Van Citters RL, Kemper WS, Franklin DL. Blood pressure responses of wild giraffes studied by radio telemetry. Science 1966;152:384-6.

26. Østergaard KH, Baandrup UT, Wang T, et al. Left Ventricular Morphology of the Giraffe Heart Examined by Stereological Methods. The Anatomical Record 2013;296:611-21.

27. Smerup M, Damkjær M, Brøndum E et al. The thick left ventricular wall of the giraffe heart normalises wall tension, but limits stroke volume and cardiac output. The Journal of Experimental Biology 2016;219:457-63.

28. Aalkjær C, Wang T. The remarkable cardiovascular system of the giraffe. Annual Review of Physiology 2021, in press.

29. Agaba M., Ishengoma E, Miller W et al. Giraffe genome sequence reveals clues to its unique morphology and physiology. Nature Communications 2016;7:11519.

30. Hirt MN, Sörensen NA, Bartholdt LM et al. Increased afterload induces pathological cardiac hypertrophy: A new in vitro model. Basic Research in Cardiology 2012;107. https://doi.org/10.1007/s00395-012-0307-z

31. Letunic I, Bork P. Interactive Tree Of Life (iTOL) v4: Recent updates and new developments. Nucleic Acids Research, 2019;47:W256-W259.

32. Williams EM. Giraffe Stature and Neck Elongation: Vigilance as an Evolutionary Mechanism. Biology 2016;5(3). https://doi.org/10.3390/biology5030035

33. Meijler FL, Meijler TD. Archetype, adaptation and the mammalian heart. Netherlands Heart Journal 2011;19:142-8.

34. Smerup M, Agger P, Nielsen EA, et al. Regional and Epi- to Endocardial Differences in Transmural Angles of Left Ventricular Cardiomyocytes Measured in Ex Vivo Pig Hearts: Functional Implications. Cardiovascular Biology 2013;296:1724-1734.

35. Omann C, Agger P, Bøgh N, et al. Resolving the natural myocardial remodelling brought upon by cardiac contraction; a porcine ex-vivo cardiovascular magnetic resonance study of the left and right ventricle. Journal of Cardiovascular Magnetic Resonance 2019;21:35.

36. Heinzel FR, Hohendanner F, Jin G et al. Myocardial Hypertrophy and Its Role in Heart Failure with Preserved Ejection Fraction. Journal of Applied Physiology 1985 (republished 2015);19:1233-42.

37. Hubel TY, Golabek KA, Rafiq K, et al. Movement patterns and athletic performance of leopards in the Okavango Delta. Proceedings of the Royal Society B: Biological Sciences 2018;285. https://doi.org/10.1098/rspb.2017.2622 
38. Brilla CG, Reams GP, Maisch B, Weber, KT. Renin-angiotensin system and myocardial fibrosis in hypertension: Regulation of the myocardial collagen matrix. European Heart Journal 1993; 14(Suppl J):57-61.

39. National Center for Biotechnology Information. PubChem Compound Summary for CID 172198, Angiotensin II. https://pubchem.ncbi.nlm.nih.gov/compound/172198 (10 August 2020, date last accessed)

40. Murphy AM, Wong, AL, Bezuhly M. Modulation of angiotensin II signaling in the prevention of fibrosis. Fibrogenesis \& Tissue Repair 2015;8. https://doi.org/10.1186/s13069-015-0023-z

41. Riordan JF. Angiotensin-I-converting enzyme and its relatives. Genome Biology 2003;4:225.

42. Baccouche BM, Natterson-Horowitz B. Giraffe Myocardial Hypertrophy as an Evolved Adaptation and Natural Animal Model of Resistance to Diastolic Heart Failure in Humans [Abstract]. International Society for Evolution, Medicine, and Public Health Annual Meeting in Zurich, Switzerland on 15 August 2019. https://easychair.org/smartprogram/ISEMPH2019/2019-08-15.html\#talk:108846

43. Brøndum E, Hasenkam JM, Secher NH et al. Jugular venous pooling during lowering of the head affects blood pressure of the anesthetized giraffe. American Journal of Physiology. Regulatory, Integrative and Comparative Physiology 2009;297: R1058-65.

44. Damkjaer M, Wang T, Brøndum E et al. The giraffe kidney tolerates high arterial blood pressure by high renal interstitial pressure and low glomerular filtration rate. Acta Physiologica (Oxford, England) 2015;214:497-510.

45. Goetz RH. Preliminary observations on the circulation in the giraffe. Trans Am Coll Cardiol 1955;5:239-48.

46. Goetz RH, Budtz-Olsen O. Scientific safari-the circulation of the giraffe. S Afr Med J 1955;29:773-6.

47. Goetz RH, Keen EN. Some aspects of the cardiovascular system in the giraffe. Angiology $1957 ; 8: 542-64$.

48. Van Citters RL, Franklin DL, Vatner SF, et al. Cerebral hemodynamics in the giraffe. Trans Am Coll Cardiol 1969;82:293-304.

49. Van Citters RL, Kemper WS, Franklin DL.. Blood flow and pressure in the giraffe carotid artery. Comp Biochem Physiol 1968;24:1035-42.

50. White CR, Seymour RS. The role of gravity in the evolution of mammalian blood pressure. Evol Int J Org Evol 2014;68:901-8.

51. Hargens AR, Millard RW, Petterson K, et al. Transcapillary fluid balance in giraffe. In Interstitial-lymphatic Liquid and Solute Movement, ed. ND Staub, JC Hogg, AR Hargens. Basel, Switz.: Karger, 1987:195-292.

52. Hargens AR, Millard RW, Pettersson K, et al. Gravitational haemodynamics and oedema prevention in the giraffe. Nature 1987;329:59-60.

53. Hargens AR, Petterson K, Millard RW. Giraffe cardiovascular adaptations to gravity. In Endothelial Biomedicine, ed. WC Aird. Cambridge UK: Cambridge University Press, 2007:94-101.

54. Mitchell G, Skinner JD, Rickards I, et al. 1990. Giraffe cerebrovascular resistance. South Afr J Sci 1990;86:1.

55. Mitchell GS, Skinner JD. How giraffe adapt to their extraordinary shape. Trans $R$ Soc South Afr 1993;48:207-18. 
56. Warren JD, Aitken-Palmer C, Weldon AD et al. Congestive Heart Failure Associated with Pregnancy in Okapi (Okapia Johnstoni). Journal of Zoo and Wildlife Medicine 2017;48:179-88.

57. Beaufrère H. Avian Atherosclerosis: Parrots and Beyond. Journal of Exotic Pet Medicine 2013;22:336-347.

58. O'Rourke MF, Avolio AP, Nichols WW. The kangaroo as a model for the study of hypertrophic cardiomyopathy in man. Cardiovascular Research 1986;20:398-402.

59. King LJ. Veterinary Medicine and Public Health. Morbidity and Mortality Weekly Report (CDC) 2006;55:7-9. 